\title{
Synthesis of well-defined functional crystals by high temperature gas-phase reactions
}

\author{
Hai-Bo Jiang $\cdot$ Lin-Feng Pan $\cdot$ Peng-Fei Liu \\ Wen-Qi Fang $\cdot$ Hua-Gui Yang
}

Received: 31 August 2013/ Accepted: 6 November 2013/Published online: 26 March 2014

(c) Science China Press and Springer-Verlag Berlin Heidelberg 2014

\begin{abstract}
Over the past decades, there have been many synthesis methods on producing well-defined crystals, due to their enormous application potentials in industrial field. Among them, high temperature gas-phase reactions (HTGR) approach may be one of the most promising processes for fabrication of well-defined crystals with controllable structure, size, shape, and composition. This review is focused on the recent progresses in synthesizing well-defined crystalline $\mathrm{TiO}_{2}$ dominated with, respectively, $\{001\}$ facets and $\{105\}$ facets, one-dimensional $\mathrm{ZnO}$ and $\mathrm{SnO}_{2}$ nanorods/nanowires, $\mathrm{MoS}_{2}$ nanosheets as well as $\mathrm{GaP}$, InP, and GaAs nanowires via HTGR approach. Although these research works were currently carried out on experimental scale, it is worth to note that the industrial importance of this HTGR approach for design and fabrication of well-defined crystals in the future owing to its advantages of continuous and scalable production with controlled dimensions and low cost.
\end{abstract}

Keywords Continuous synthesis - Well-defined crystals $\cdot$ High temperature gas-phase reactions

SPECIAL ISSUE: Advanced Materials for Clean Energy

H.-B. Jiang · L.-F. Pan · P.-F. Liu · W.-Q. Fang · H.-G. Yang $(\triangle)$

Key Laboratory for Ultrafine Materials of Ministry of Education, School of Materials Science and Engineering, East China University of Science and Technology, Shanghai 200237, China e-mail: hgyang@ecust.edu.cn

H.-G. Yang

Centre for Clean Environment and Energy, Griffith University, Gold Coast Campus, Brisbane, QLD 4222, Australia

\section{Introduction}

Well-defined inorganic functional crystals with tailor-made crystal facets have attracted great research interest owing to their realistic applications in catalysis, sensors, batteries, and environmental remediation [1-8]. Unfortunately, the surfaces with high reactivity usually diminish rapidly during the crystal growth process as a result of the minimization of surface energy. Thus, increasing the percentage of known highly reactive surfaces or creating new favorable surfaces is highly desirable. In recent years, a number of hydro-/solvothermal and HTGR have been extensively studied, to control the growth rate of different crystal faces and subsequently tailor the exposed facets [9-14]. Compared to wet chemical methods, HTGR method has several unique merits, (1) the sample can be produced with continuous or semi-continuous operation; (2) high temperature provides the product with controlled scale dimensions and a high degree of crystallinity; and (3) a remarkable growth rate; which can be expected to substantially reduce the cost of producing well-defined crystals on an industrial scale. Although HTGR has been applied sporadically in industrial production to manufacture fumed silica (Cabot Corporation, USA) and titanium dioxide (DuPont Company, USA) since 1940s and 1950s, respectively, mass production of well-defined single crystals via the HTGR process is still a big challenge, considering the fact that the time of chemical reaction and crystal growth during the HTGR process is very short only about several seconds [13-23].

In recent years, this situation has changed that many well-defined single crystals, such as metal oxide, metal sulfide, and group III-V compounds, have been successionally synthesized in some special designed novel HTGR reactors. For the present writing, we aim to provide a brief overview of the growth mechanism in HTGR and specific 
details on the preparations and properties of materials mentioned above. At the end of this review, some outlook and perspectives will also be given to illustrate the opportunities and challenges in the field of HTGR.

\section{Preparation and enhanced properties of well-defined crystals}

\subsection{Formation mechanism of well-defined crystals via HTGR process}

A typical schematic reaction flow chart is given in Fig. 1a. In an experiment, liquid or solid reactants evaporated in a vaporization chamber, and then were carried into the high temperature region by inert gas $\left(\mathrm{Ar}\right.$ or $\left.\mathrm{N}_{2}\right)$. At the same time, another reactant gas adjusted by gas mass controller went through the reactor. The final products were collected downstream by a bag filter. Both electricity and fuel could be used as heat source to provide the high temperature in reaction areas. The formation of well-defined crystals in HTGR can be roughly divided into two distinct stages: nucleation and growth of seeds into crystals, as illustrated in Fig. 1b.

Nucleation is the very early stage of any crystallization process. As the concentration of precursor molecules become sufficiently high in gas, they start to gather into nanometer scale clusters and then constitute the primary nuclei through homogeneous nucleation. With a continuous supply of the building blocks such as atoms, ions, and product molecules, the nuclei can serve as seeds for further growth to form the critical crystallite size.

In fact, the morphology and shape of the final product are controlled by interplay between growing thermodynamics and kinetics. Typically, the thermodynamic equilibrium shape of the most stable product will be produced according to Gibbs-Wulff theorem. However, in many growing process final crystals dominated by high-energy facets could be obtained by tuning the reaction conditions before the reaction reaches equilibrium. That is to say, kinetic factor plays a key role in crystal growth which enlarges the percentage of high-energy surfaces and makes the final products to be different from the thermodynamically favored shapes. Other factors, such as the concentration of precursors, mass transport limitation, and modification of the surface energy realized by adsorption of additives or impurities, can also alter the crystal growth habit by tuning the relative growth rates of the different facets. As a result, it is very important to design suitable experimental conditions and reactors in order to synthesize the desired crystals bounded with high-energy facets. Some representative studies related to fabrication of crystals bounded with particular facets by HTGR method are summarized in the next section.

\subsection{Preparation and enhanced properties of metal oxide well-defined crystals}

Well-defined crystalline metal oxides are the research hot spot in many promising applications, such as catalysts, gas sensors, electronic, optoelectronic, and thermoelectric devices [13, 14, 24-30]. In this section, the preparations of $\mathrm{TiO}_{2}, \mathrm{ZnO}$, and $\mathrm{SnO}_{2}$ via HTGR process and their properties are reviewed.

Crystalline $\mathrm{TiO}_{2}$ in the anatase phase is widely used as photocatalyst in energy generation and environmental treatment, owing to its low cost, non-toxic feature as well as compatible photoactivity under ultraviolet irradiation. Conventionally, anatase $\mathrm{TiO}_{2}$ crystals are dominated by the thermodynamically stable $\{101\}$ facets (ca. $94 \%$, according to the Wulff construction) and a minority of high activity $\{001\}$ facets. Recently, in order to increase percentage of highly reactive $\{001\}$ facets, various capping agents were employed to synthesize desirable well-defined crystals in hydrothermal conditions. However, hydrothermal process has many intrinsic disadvantages such as long reaction time, high production cost, and low yield [9]. a

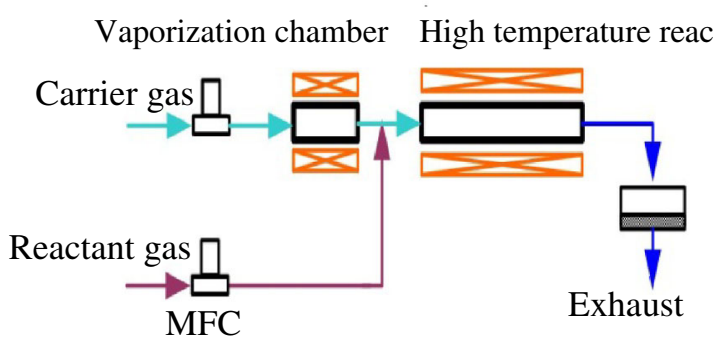

b

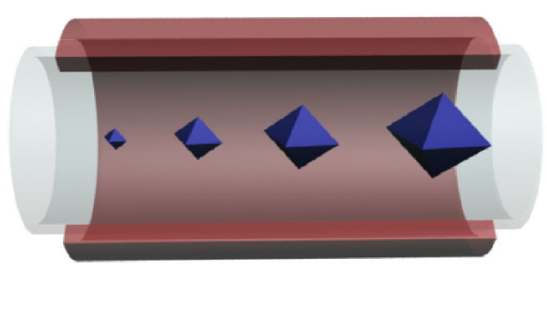

Fig. 1 (Color online) Schematic experimental apparatus for the production of well-defined crystals (a) and schematic diagram of growth mechanism for well-defined crystals (b) 
To overcome the shortcomings of hydrothermal process, Ohtani and co-workers [13] (using a special designed reactor) synthesized the anatase $\mathrm{TiO}_{2}$ with dominated $\{001\}$ facets via modified HTGR method. This method only needs very short reaction time of several seconds and the detailed procedure is presented as following. The vapor of $\mathrm{TiCl}_{4}$ was liberated by bubbling $\mathrm{Ar}(200 \mathrm{~mL} / \mathrm{min})$ into a $\mathrm{TiCl}_{4}$ solution at $358 \mathrm{~K}$, mixing it with an $\mathrm{O}_{2}$ stream $(1,200 \mathrm{~mL} / \mathrm{min})$, and fed into a quartz glass tube heated from the outside by an oxyhydrogen flame burner. The tube was rotated around the cylindrical axis at a speed of $55 \mathrm{r} /$ min for homogeneous heating. The heating temperature was adjusted to $1,573 \mathrm{~K}$. The product single crystals images are shown in Fig. 2.

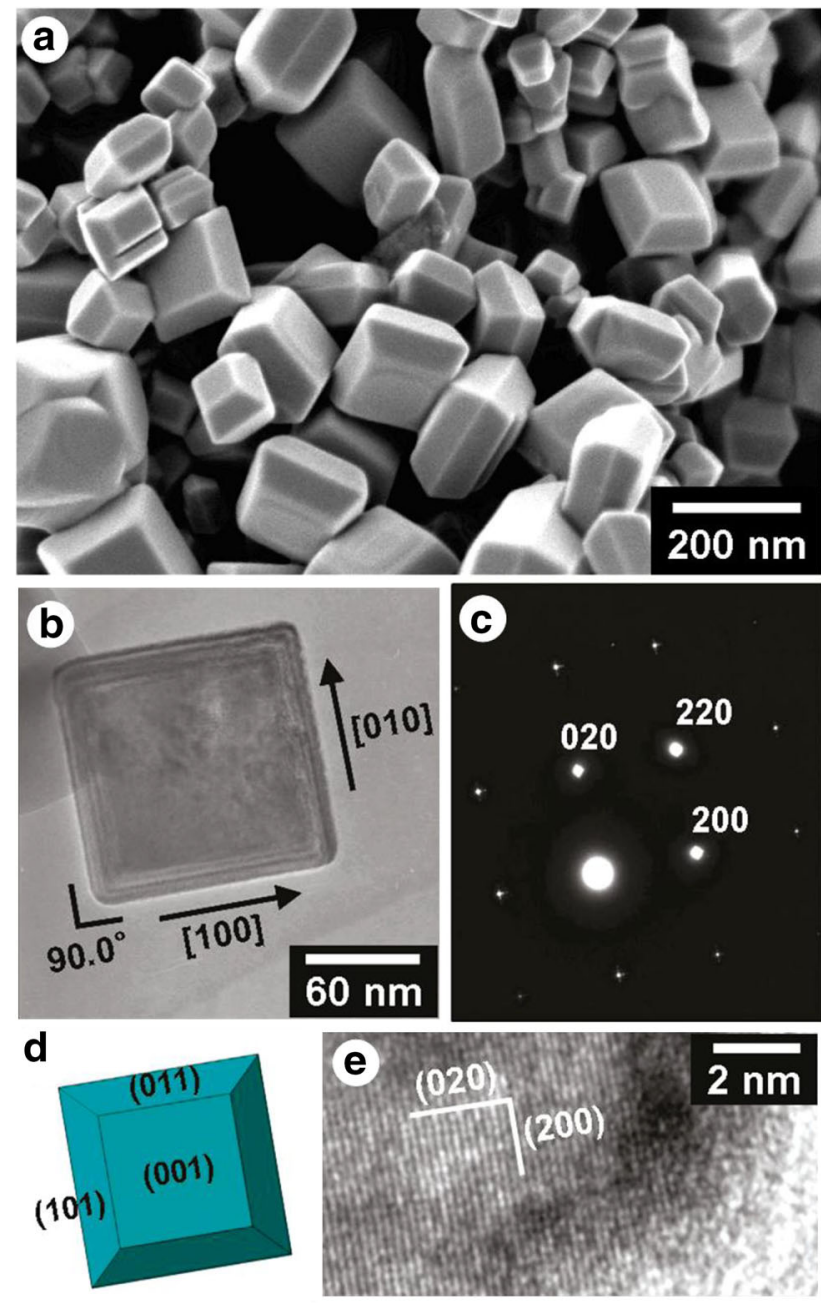

Fig. 2 (Color online) Scanning electron microscope (SEM) image of $\mathrm{TiO}_{2}$ particles prepared by gas-phase reaction of $\mathrm{TiCl}_{4}$ with oxygen (a), transmission electron microscope (TEM) image (b), electron diffractions (c), schematic shape (d), and high resolution TEM image (e) of decahedral $\mathrm{TiO}_{2}$ particles recorded along the $c$-axis. Reprinted with permission from Ref. [13], Copyright (2009) ACS
The authors concluded that uniform and rapid heating at a high temperature would enable homogeneous nucleation and subsequent growth to well-faceted crystals with few defects. The low concentration of $\mathrm{TiCl}_{4}$ and the narrow heating zone would prevent formation of large particles and polycrystalline aggregates with grain boundaries. The photocatalytic activity of the well-defined crystal was higher than that of commercialized P25 under various conditions, though the specific surface area of well-defined crystal is less than a third of the P25 $\left(9.4 \mathrm{~m}^{2} / \mathrm{g}, \mathrm{P} 25: 48 \mathrm{~m}^{2} /\right.$ g). HTGR environment could provide the well-defined photocatalyst with low density of crystalline defects, which might be the reason for the extremely high photocatalytic activity for $\mathrm{H}_{2}$ evolution from an aqueous methanol solution and oxidative decomposition of organic compounds in an aqueous solution.

Not only the anatase $\mathrm{TiO}_{2}$ dominated with $\{001\}$ facets but also anatase $\mathrm{TiO}_{2}$ exposed with high-index crystal planes were explored by HTGR method [14]. Recently, we successfully used HTGR to synthesize anatase $\mathrm{TiO}_{2}$ crystals with predominantly exposed high-index $\{105\}$ facets, which is depicted as following. A straight static furnace pipe and a thin spiral tube were used as reactor and reactant feeder, respectively. The vapor-phase $\mathrm{TiCl}_{4}$ was liberated by bubbling $\mathrm{O}_{2}$ into $\mathrm{TiCl}_{4}$ liquid and then passed through the furnace pipe. The experimental process was shown to be quite robust, reproducible (as shown in Fig. 3). The theoretical calculation results suggest that $\mathrm{H}_{2} \mathrm{O}$ can only adsorb along the step edges dissociatively, while it is unable to stay at the flat facet. The adsorption energy was estimated to be $1.03 \mathrm{eV}$ under the local coverage of $1 / 2$ (with respect to the edge $\mathrm{Ti}$ or $\mathrm{O}$ ). In another word, the $\{105\}$ facets should have the capability to cleave water photocatalytically and this prediction was confirmed by experiment.

Beside the preparation of anatase $\mathrm{TiO}_{2}$ exposed with specific facets via HTGR process, one-dimensional (1D) metal oxides could be synthesized by this method as well. Han et al. [24] successfully synthesized $\mathrm{ZnO}$ nanorods and the results suggested that nanorods with the length of $500 \mathrm{~nm}$ showed higher gas sensing property than 200 and 2,000 nm length ones. Chang et al. [25] used HTGR process modified with vapor trapping method to synthesize n-type $\mathrm{ZnO}$ nanowires which shows high carrier concentration without incorporating impurity dopants. Furthermore, dopant may also be introduced during the HTGR process to control the shape of well-defined crystals. Height et al. [26] found that In and Sn dopants could progressively alter the shape of the $\mathrm{ZnO}$ particles to a rodlike shape with increasing dopant concentration. Meanwhile, Liu et al. [30] synthesized single-crystalline $\mathrm{SnO}_{2}$ nanorods via HTGR method by introducing Fe as dopant as well. The as-prepared $\mathrm{SnO}_{2}$ nanorods with uniform length 

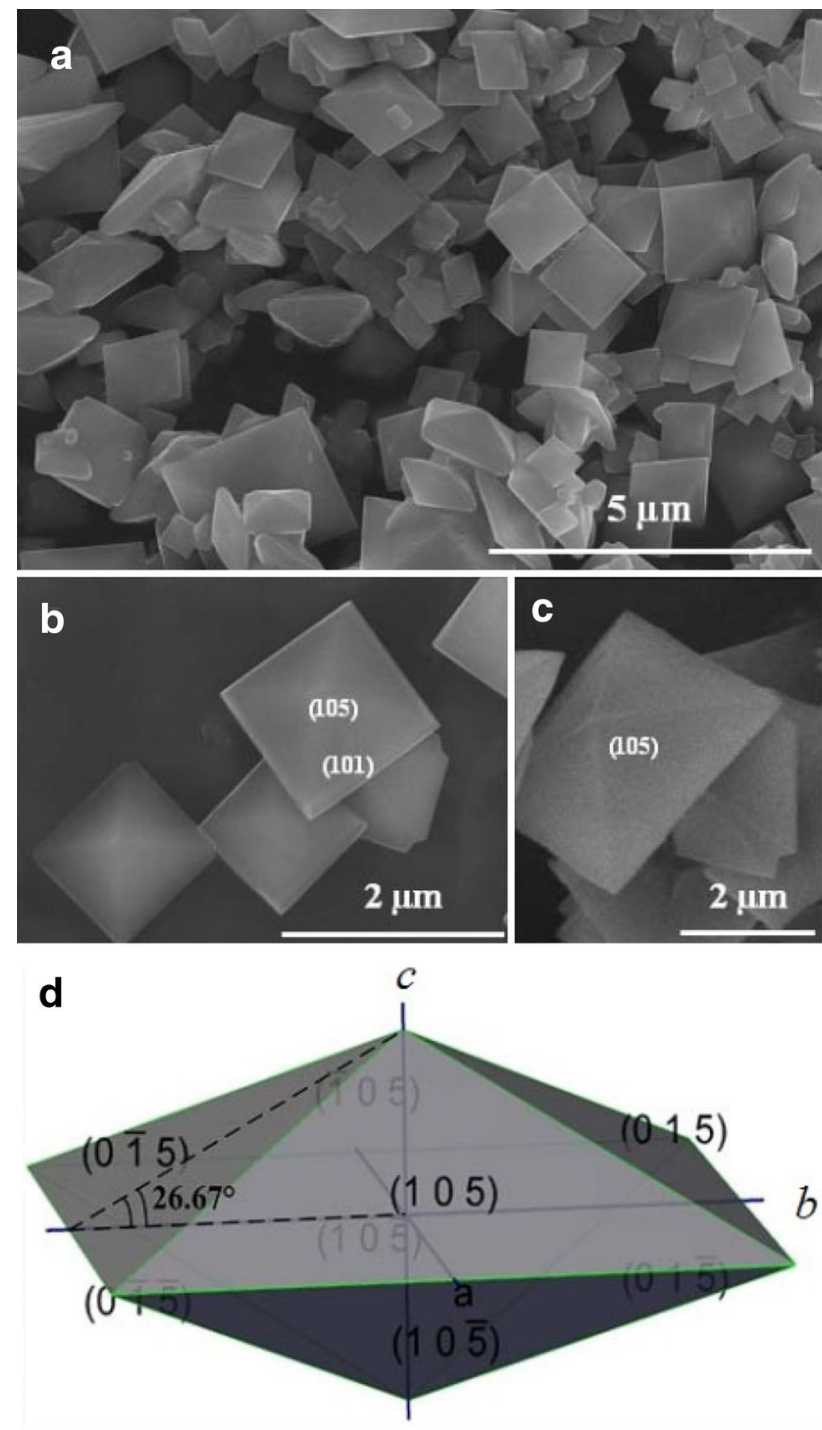

Fig. 3 (Color online) a-c SEM images and $\mathbf{d}$ schematic shape of the as-obtained anatase $\mathrm{TiO}_{2}$ crystals dominated by high-index $\{105\}$ facets. Reproduced from Ref. [14], Copyright (2011) Wiley

up to $200 \mathrm{~nm}$ and diameter around $20 \mathrm{~nm}$ are smooth and single crystal rutile structures, growing along the [001] direction. The typical TEM images and the corresponding selected area electron diffraction (SAED) pattern are given in Fig. 4. It is noteworthy that this dopant-assisted HTGR approach provides a new strategy for sequentially engineering 1D nanomaterials.

\subsection{Preparation and their properties of chalcogenides}

The chalcogenides are binary compounds of metals and the chalcogenides (sulfur, selenium, or tellurium), which display important properties and potential for diverse applications in catalysis [31-33], lubrication [34], field effect transistors (FET) materials [35, 36], sensing [37], and energy conversion and storage [38, 39]. Particularly, 2D

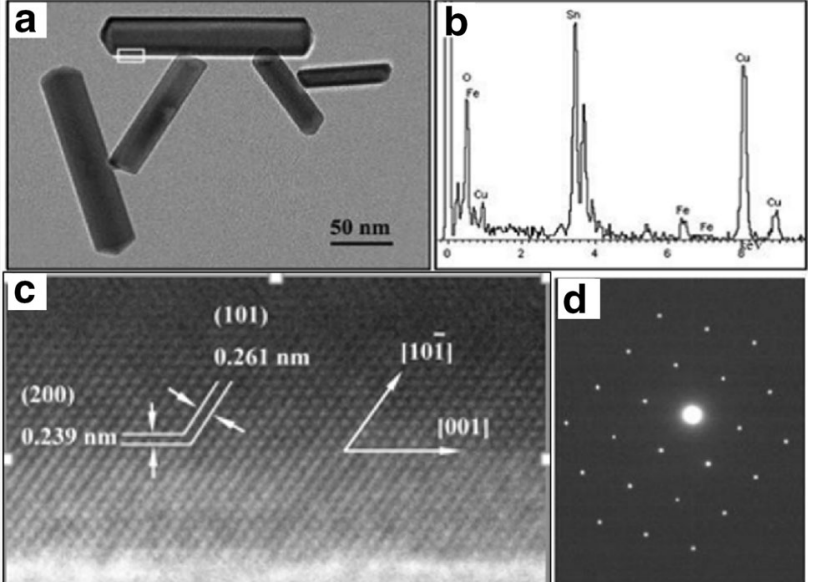

Fig. 4 a TEM image of 2.5 at.\% Fe-doped $\mathrm{SnO}_{2}$ nanorods, and bd energy disperse spectroscopy (EDS) analysis, HRTEM image, and corresponding SAED pattern taken from the white box in a, showing the preferred [001] orientation. Reprinted with permission from Ref. [30], Copyright (2010) ACS

metal dichalcogenides with graphite-like-layered structures show outstanding properties in industrial catalysis and have attracted significant attention in the last 20 years. Some recent progresses on their HTGR synthesis processes are reviewed in this section.

Most of the current researches of HTGR process have been focused on $\mathrm{MoS}_{2}$ which is also the testing ground for experiments in monolayers of metal dichalcogenides [40]. In the case of single-source precursor reaction, ammonium thiomolybdate $\left.\left[\left(\mathrm{NH}_{4}\right)_{2} \mathrm{MoS}_{4}\right)\right]$ is often used. It has been reported that the thermolysis of $\left(\mathrm{NH}_{4}\right)_{2} \mathrm{MoS}_{4}$ in a dynamic inert atmosphere $\left(\mathrm{N}_{2}\right)$ results in the conversion of $\left(\mathrm{NH}_{4}\right)_{2} \mathrm{MoS}_{4}$ to $\mathrm{MoS}_{3}$ in the temperature region 120 $260{ }^{\circ} \mathrm{C}$ [41], and another annealing is needed to convert $\mathrm{MoS}_{3}$ to $\mathrm{MoS}_{2}$.

$\left(\mathrm{NH}_{4}\right)_{2} \mathrm{MoS}_{4} \rightarrow 2 \mathrm{NH}_{3}+\mathrm{H}_{2} \mathrm{~S}+\mathrm{MoS}_{3}$

$\left(\mathrm{NH}_{4}\right)_{2} \mathrm{MoS}_{4}+\mathrm{H}_{2} \rightarrow 2 \mathrm{NH}_{3}+2 \mathrm{H}_{2} \mathrm{~S}+\mathrm{MoS}_{2}$

Shi et al. [42] demonstrated the van der Waals epitaxy of $\mathrm{MoS}_{2}$ layers on a chemical vapor deposition (CVD) grown graphene surface (Fig. 5a). They use dimethyl formamide (DMF) as the solvent and argon as the carrier gas to obtain the $\mathrm{MoS}_{2} / \mathrm{CVD}$ graphene hybrid in a low-pressure HTGR system. The $\mathrm{MoS}_{2}$ flakes forming initially from the graphene wrinkles (Fig. 5b) tend to have a hexagonal shape with $c$-axis perpendicular to the graphene surface. Notice that the lattice spacing for $\mathrm{MoS}_{2}$ is $28 \%$ larger than that for graphene, it is relaxed through the weak wan der Waals force. The same precursor is also reported for selective solvothermal synthesis of $\mathrm{MoS}_{2}$ nanoparticles and deposition of three-layered $\mathrm{MoS}_{2}$ sheets by dip-coating $[31,43]$. 


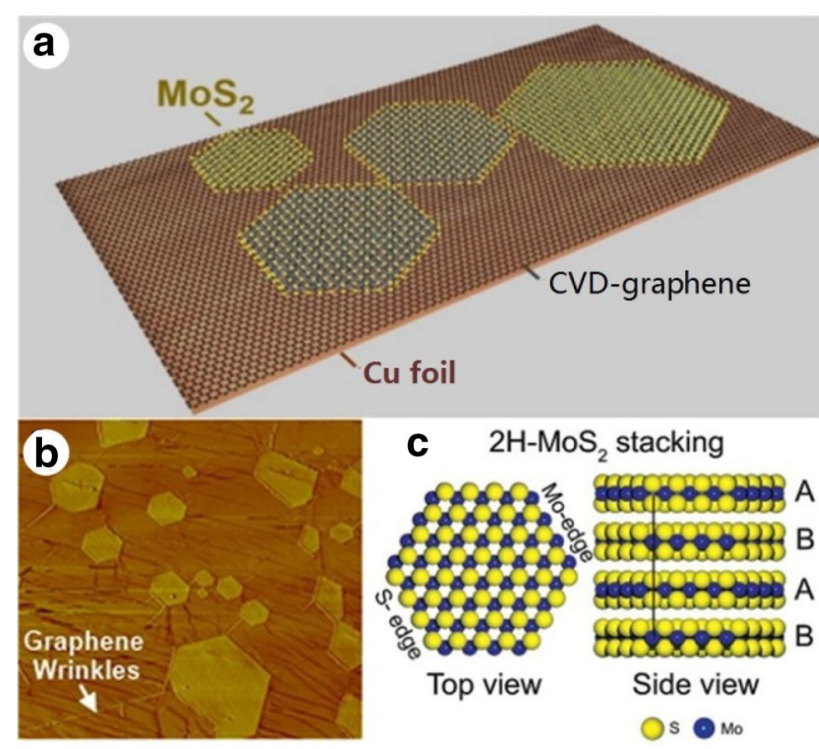

Fig. 5 (Color online) $\mathrm{MoS}_{2}$ nanosheets on CVD graphene/Cu substrates through van der Waals epitaxy $(\mathbf{a}, \mathbf{b})$ and ball model of $2 \mathrm{H}-\mathrm{MoS}_{2}$ with Mo- and S-edges successively being exposed (c). Reproduced with permission from: a, b Ref. [42] and c Ref. [45], Copyright (2012) ACS

Besides the common form hexagonal molybdenum sulfide, the shapes of most direct experimental $\mathrm{MoS}_{2}$ are triangular nanosheets. The $\mathrm{MoS}_{2}$ nanocrystallites with (0001) basal plane being oriented parallel to the substrate surface was prepared by depositing Mo on $\mathrm{Au}$ (111) in a $\mathrm{H}_{2} \mathrm{~S}$ atmosphere followed by annealing [44]. Ultrahigh vacuum scanning tunneling microscopy (STM) was used to study the edges structure of the well-defined crystals. Combined with density functional theory (DFT) calculations, it was verified that the Mo-edge with $\mathrm{S}$ atoms terminated the triangular nanoclusters, which are shifted by half a lattice constant relative to the $\mathrm{S}$ atoms in the basal plane.

Subsequently, Besenbacher and co-workers [45] deposited Mo onto a defective highly oriented pyrolytic graphite (HOPG) in a sulfiding $\mathrm{H}_{2} \mathrm{~S}$ atmosphere corresponding to $5 \times 10^{-6}$ mbar $(1 \mathrm{mbar}=100 \mathrm{~Pa})$ and followed by an annealing treatment to facilitate full sulfidation and crystallization. The $\mathrm{MoS}_{2}$ shows a hexagonally truncated shape relative to the former one on $\mathrm{Au}$ (111). This was attributed to a lower chemical potential of sulfur which may change the relative stability of the two types of edges, exposing both (1010) Mo-edge and the (1010) S-edge (Fig. 5c). Either edge is believed to be catalytically important. Jaramillo et al. [46] identified the active sites for catalysis of hydrogen evolution reaction (HER). They proved experimentally that the sulfide-terminated Mo-edge is the dominant edge structure in triangular $\mathrm{MoS}_{2}$. The calculation results suggest that only 1 in 4 edge atoms can evolve molecular $\mathrm{H}_{2}$ at a given time.
Zhan et al. [47] demonstrated a scalable fabrication of $\mathrm{MoS}_{2}$ on $\mathrm{SiO}_{2}$ substrates. Pure sulfur was placed in the upwind zone set at the temperature a little above the melting point $\left(113{ }^{\circ} \mathrm{C}\right)$ while Mo thin film that was predeposited on the $\mathrm{SiO}_{2}$ substrate which was placed in the center of a quartz tube furnace. The tube was first kept in a flowing protective atmosphere of high-purity $\mathrm{N}_{2}$ and then the temperature was gradually increased to $500{ }^{\circ} \mathrm{C}$ in $30 \mathrm{~min}$. Finally, the tube was heated to $750{ }^{\circ} \mathrm{C}$ over $90 \mathrm{~min}$ and was kept at $750{ }^{\circ} \mathrm{C}$ for $10 \mathrm{~min}$ before being cooled down to room temperature. The resistivity of their samples are from $-1.46 \times 10^{4}$ to $2.84 \times 10^{4} \Omega / \mathrm{cm}^{2}$, about two orders of magnitude higher than CVD graphene (125 $\Omega$ / $\mathrm{cm}^{2}$ ). In addition, the mobility at room temperature of the as grown $\mathrm{MoS}_{2}$ sheets range from 0.004 to $0.04 \mathrm{~cm}^{2} /(\mathrm{V} \mathrm{s})$ which was much lower than mechanically exfoliated $\mathrm{MoS}_{2}$ flakes. They found that the size and thickness of the atomic $\mathrm{MoS}_{2}$ layer only depend on the size of the substrate and the thickness of the pre-deposited Mo.

Lee et al. [48, 49] have reported an alternative method for synthesizing large-area $\mathrm{MoS}_{2}$ ultrathin films using the gas-phase reaction of $\mathrm{MoO}_{3}$ and $\mathrm{S}$ powder. Since the growth of $\mathrm{MoS}_{2}$ is very sensitive to the substrate surface, perylene-3,4,9,10-tetracarboxylic acid tetrapotassium salt or perylene-3,4,9,10-tetracarboxylic dianhydride was usually used to modify the substrates prior to the growth. They showed that promoted growth can be achieved using the sulfurization of $\mathrm{MoO}_{3}$ and the mobility of $\mathrm{MoS}_{2}$ on $\mathrm{SiO}_{2}$ substrate is similar to the value reported by Zhan et al. [47].

Despite substantial work on synthesis of $\mathrm{MoS}_{2}$ via HTGR has performed, other materials of the most interest including chalcogenides of group III (GaSe, GaTe, InSe), group IV ( $\mathrm{GeS}, \mathrm{GeSe}, \mathrm{SnS}, \mathrm{SnSe}, \mathrm{GeSe}_{2}$ ), and transition metal dichalcogenides $\left(\mathrm{TiS}_{2}, \mathrm{WS}_{2}\right)$ have attracted considerable attention [50-56]. These as-described methods usually contain a sublimation of metal and chalcogen precursors followed by deposition onto a substrate. Sulfurization (or selenization) of metal or its precursors can happen before or after the deposition. Whether the gasphase reaction is catalyst-free or not, choosing suitable substrate, deposition temperature, and source materials may achieve control of products easily. Because of the requirement of suitable substrates in the preparation of chalcogenides, this process may be considered as semicontinuous operation mode.

\subsection{The growth of group III-V compounds}

Metal phosphides have been widely used in optoelectronic devices, magnetic storage devices, magnetic refrigeration systems, industrial catalysis, and battery materials due to their special structures and properties. Therefore, several techniques have been developed to fabricate metal 
phosphides with well-defined facets through high temperature gas-phase reactions [57-59]. Most of these high temperature reactions are usually used to get $1 \mathrm{D}$ semiconductor structures. These HTGR processes usually need an anaerobic condition which prevents synthesized phosphides from oxidation, and often involve gold particles as catalysts to direct the 1D structure growth by the vaporliquid-solid (VLS) mechanism [60-62].

During the fabrication of $\mathrm{GaP}$ and $\mathrm{InP}$ nanowires by HTGR process, a special reactor was used to provide a lowpressure and high temperature environment for nanowires growth. In the reactor cell, metal-organic sources reacted with $\mathrm{PH}_{3}$. The target products grew on the substrates $(\mathrm{GaP}$ substrate and InP substrate are used, respectively) deposited with gold droplets [63]. Prior to the reaction, $\mathrm{PH}_{3}$ flow was first used to remove the oxides or organics on the substrate at the reaction temperature. Then, the metalorganics (trimethylgallium or trimethylindium) and phosphine $\left(\mathrm{PH}_{3}\right)$ acted as precursors with a specific molar ratio which were carried by $\mathrm{H}_{2}$ at a specific flow rate went into the high temperature reactor. Sometimes, hydrogen chloride was used to suppress the overgrowth of the nanowires [64]. Recently, Assali et al. [65] have successfully synthesized the GaP nanowire arrays (shown in Fig. 6) with pure hexagonal crystal structure and the direct nature of the band gap, which enables new pathways to tailor materials properties and enhancing the functionality. Algra et al. [66] first demonstrated that the crystal structure of InP nanowires could be controlled by using impurity dopants. Meanwhile, Piret et al. [67] have got perfectly vertical GaP
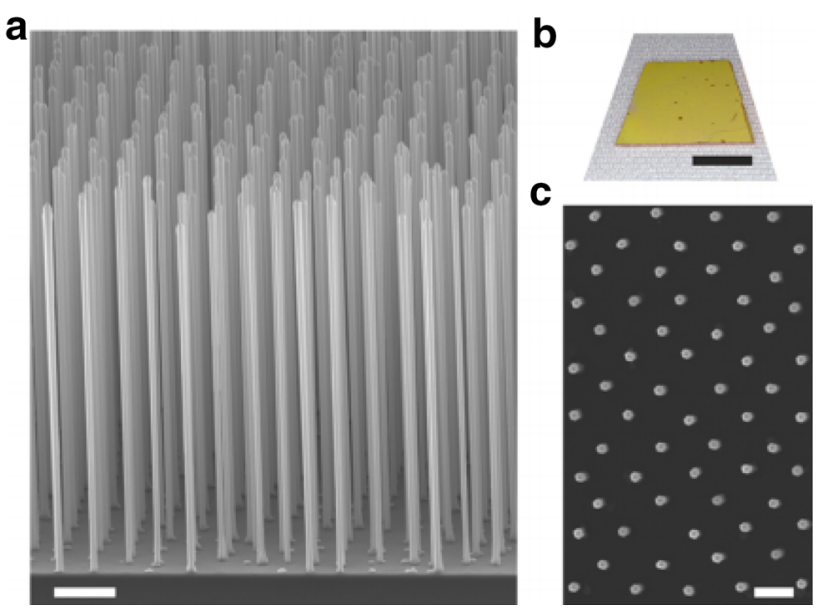

Fig. 6 (Color online) Uniformity of GaP nanowires arrays. a SEM picture of $\mathrm{GaP} / \mathrm{Al}_{0.4} \mathrm{Ga}_{0.6} \mathrm{P}$ core/shell nanowires in a nanoimprint pattern. b Optical image of the nanowires sample. Scale bar corresponds to $0.5 \mathrm{~cm}$. c SEM topview of the same sample as in a showing the periodicity of the nanoprint pattern. Scale bar corresponds to $500 \mathrm{~nm}$. Reprinted with permission from Ref. [65], Copyright (2013) ACS nanowires on the substrate with monodisperse size and controllable specific geometry.

Based on the successful synthesis of long silicon nanowires by PLV method [68], GaP and InP nanowires have been successfully synthesized through this method [69]. The targets were made by GaP (or InP) powder mixed with gold powder, which were centered in a long quartz tube with an inner small quartz tube. A tube furnace was used to control the target temperature. Along with the $\mathrm{Ar} / \mathrm{H}_{2}$ flow, the target was ablated and vaporized by the laser beam for about $2 \mathrm{~h}$. Then, the reaction system was slowly cooled down to the room temperature. A light yellow product was achieved on the wall of the quartz tube.

Thermal evaporation is another HTGR to synthesize metal phosphides. Red phosphorus is often used as the precursor instead of phosphine $\left(\mathrm{PH}_{3}\right)$. Before the reaction, metal, metal oxides, and red phosphorus should be milled for about several minutes. A tube furnace was used to supply a high temperature reaction environment (usually above $900{ }^{\circ} \mathrm{C}$ ). Prior to heating, Ar flow was used to remove oxygen in the tube furnace. $\mathrm{Al}_{2} \mathrm{O}_{3}$ [59], $\mathrm{Si}$ [70], and AlN [71] were often used as substrates for nanowires growth. Through this process, $\mathrm{Ga}, \mathrm{Ga}_{2} \mathrm{O}_{3}$, and red phosphors reacted at high temperature and $\mathrm{GaP}$ nanotubes were achieved by $\mathrm{Wu}$ et al. [71]. The growth mechanism and process for the formation of $\mathrm{GaP}$ nanotubes were explained as follows [72, 73]:

$$
\begin{aligned}
& 4 \mathrm{Ga}(\mathrm{l})+\mathrm{Ga}_{2} \mathrm{O}_{3}(\mathrm{~s}) \rightarrow 3 \mathrm{Ga}_{2} \mathrm{O}(\mathrm{g}) \\
& 3 \mathrm{Ga}_{2} \mathrm{O}(\mathrm{g})+2 \mathrm{AlN}(\mathrm{s}) \rightarrow \mathrm{Al}_{2} \mathrm{O}_{3}(\mathrm{~s})+6 \mathrm{Ga}(\mathrm{l})+\mathrm{N}_{2}(\mathrm{~g}) \\
& \mathrm{P}(\mathrm{g})+\mathrm{Ga}(\mathrm{l}) \rightarrow \mathrm{GaP}(\text { nanotube })
\end{aligned}
$$

When GaP species reached a supersaturated level, they were precipitated out from the Ga droplets. P vapor and the newly produced $\mathrm{Ga}$ species were continuously complemented into the Ga droplets to sustain this growth, and finally the GaP nanotubes were obtained. This process is similar to VLS growth [68]. Fu et al. [74] have synthesized single-crystal diameter-modulated $\mathrm{GaP}$ nanochains and apply them into nanodevices. $\mathrm{Ga}_{2} \mathrm{O}_{3}$ and red phosphorus were chosen as precursors and Si sheets treated by Shiraki method [75] was used as a substrate for the growth of $\mathrm{GaP}$ nanochains. In the high temperature, $\mathrm{Ga}_{2} \mathrm{O}_{3}$ was reduced by $\mathrm{H}_{2}$ to form $\mathrm{Ga}$ vapor, which reacted with red phosphorus to form $\mathrm{GaP}$ nanowires. Then, Ga droplets which are absorbed on the surface of GaP nanowires should form nuclei for GaP knots [76]. Because of the identical material, $\mathrm{GaP}$ prefers to grow epitaxially without misfit, consequently, the nanochains should be energetically stable. Moreover, for a large particle, spherical surface is the most effective way to reduce the surface energy of the system, so as to maintain the stability of the system. For these two reasons, the nanochains should have a very stable morphology due to their minimized system energies. 
Although GaP and InP nanowires could be fabricated by HTGR process, the requirement of the substrates limited these processes in semi-continuous operation mode. To overcome this issue, Heurlin et al. [77] have successfully designed a new HTGR reactor to continuously produce GaAs nanowires with the growth rate of $1 \mu \mathrm{m} / \mathrm{s}$. Different from the traditional, substrate-based methodology of III-V materials, they used size-selected $\mathrm{Au}$ aerosol particles as catalysts to induce the 1D growth of GaAs materials. Because of the strict control of the Au size, the nanowire lateral dimension can be sensitively tuned, so that the material properties such as quantum confinement and electron scattering which allow the optical [78] and electrical [79] properties of nanowires are fine-tuned. Through this continuous high temperature gas-reaction, high-quality nanowires could be largely produced with low cost.

\section{Summary and outlook}

This paper discusses the possible formation mechanism and selected examples of well-defined crystals synthesized via HTGR method. As an economical and efficient method, HTGR provides a powerful tool for tailoring the shapes as well as physical and chemical properties of well-defined crystals. However, only binary compounds with well-defined facets were prepared by HTGR process to date, in which exposed surfaces could be tailored by changing the experimental conditions and specific reactors. Furthermore, the formation mechanism of these binary semiconductors is not well understood. With in-depth studying on this subject, we believe that binary, ternary, even more complicated compound semiconductors with more desirable shapes, morphologies, and sizes will be synthesized by HTGR process. Although the well-defined crystals could only be produced by HTGR method on experimental scale so far, which does not meet the needs of commercial applications, it is anticipated that this method would provide new opportunities to produce the well-defined crystals on industrial scale in the future, because HTGR method can enable the mass production with perfect crystallinity, reproducibility, and controlled dimensions as well as the material composition. In addition, its continuous operation mode can also lower the cost of the final products that will benefit the commercial applications.

Acknowledgments This work was supported by the National Natural Science Foundation of China (91022023, 21076076), the Scientific Research Foundation for the Returned Overseas Chinese Scholars, State Education Ministry (SRF for ROCS, SEM), Programme for Professor of Special Appointment (Eastern Scholar) at Shanghai Institutions of Higher Learning, Major Basic Research Programme of Science and Technology Commission of Shanghai Municipality (10JC1403200), and Shanghai Municipal Natural Science Foundation (12ZR1407500).

\section{References}

1. Tian N, Zhou ZY, Sun SG et al (2007) Synthesis of tetrahexahedral platinum nanocrystals with high-index facets and high electro-oxidation activity. Science 316:732-735

2. Vittadini A, Selloni A, Rotzinger FP et al (1998) Structure and energetics of water adsorbed at $\mathrm{TiO}_{2}$ anatase (101) and (001) surfaces. Phys Rev Lett 81:2954-2957

3. Vittadini A, Casarin M, Selloni A (2007) Chemistry of and on $\mathrm{TiO}_{2}$-anatase surfaces by DFT calculations: a partial review. Theor Chem Acc 117:663-671

4. Lazzeri M, Selloni A (2001) Stress-driven reconstruction of an oxide surface: the anatase $\mathrm{TiO}_{2}(001)-(1 \times 4)$ surface. Phys Rev Lett 87:266105

5. Liu SW, Yu JG, Jaroniec M (2010) Tunable photocatalytic selectivity of hollow $\mathrm{TiO}_{2}$ microspheres composed of anatase polyhedra with exposed 001 facets. J Am Chem Soc 132:11914-11916

6. Zhang D, Li G, Yang X et al (2009) A micrometer-size $\mathrm{TiO}_{2}$ single-crystal photocatalyst with remarkable $80 \%$ level of reactive facets. Chem Commun 29:4381-4383

7. Han XG, Jin MS, Xie SF et al (2009) Synthesis of tin dioxide octahedral nanoparticles with exposed high-energy 221 facets and enhanced gas-sensing properties. Angew Chem Int Ed 48:91809183

8. Xing J, Fang WQ, Zhao HJ et al (2012) Inorganic photocatalysts for overall water splitting. Asian J Chem 7:642-657

9. Yang HG, Sun CH, Qiao SZ et al (2008) Anatase $\mathrm{TiO}_{2}$ single crystals with a large percentage of reactive facets. Nature 453:638-641

10. Yang HG, Liu G, Qiao SZ et al (2009) Solvothermal synthesis and photoreactivity of anatase $\mathrm{TiO}_{2}$ nanosheets with dominant 001 facets. J Am Chem Soc 131:4978

11. Ma XY, Chen ZG, Hartono SB et al (2010) Fabrication of uniform anatase $\mathrm{TiO}_{2}$ particles exposed by 001 facets. Chem Commun 46:6608-6610

12. Han XG, Kuang Q, Jin MS et al (2009) Synthesis of titania nanosheets with a high percentage of exposed (001) facets and related photocatalytic properties. J Am Chem Soc 131:3152-3153

13. Amano F, Prieto-Mahaney OO, Terada Y et al (2009) Decahedral single-crystalline particles of anatase titanium(IV) oxide with high photocatalytic activity. Chem Mater 21:2601-2603

14. Jiang $\mathrm{HB}, \mathrm{Cuan} \mathrm{Q}$, Wen $\mathrm{CZ}$ et al (2011) Anatase $\mathrm{TiO}_{2}$ crystals with exposed high-index facets. Angew Chem Int Ed 50:3764-3768

15. Pratsinis SE, Spicers PT (1998) Competition between gas phase and surface oxidation of $\mathrm{TiCl}_{4}$ during synthesis of $\mathrm{TiO}_{2}$ particles. Chem Eng Sci 53:1861-1868

16. Jang HD, Kim SK, Kim SJ (2001) Effect of particle size and phase composition of titanium dioxide nanoparticles on the photocatalytic properties. J Nanopart Res 3:141-147

17. Yeha CL, Yehb SH, Ma HK (2004) Flame synthesis of titania particles from titanium tetraisopropoxide in premixed flames. Powder Technol 145:1-9

18. Sun Y, Raman V, Fox RO (2011) Large-eddy-simulation-based multiscale modeling of $\mathrm{TiO}_{2}$ nanoparticle synthesis in a turbulent flame reactor using detailed nucleation chemistry. Chem Eng Sci 66:4370-4381

19. Mehta M, Sung Y, Raman V et al (2010) Multiscale modeling of $\mathrm{TiO}_{2}$ nanoparticle production in flame reactors: effect of chemical mechanism. Ind Eng Chem Res 49:10663-10673

20. Spicer PT, Chaoul O, Tsantilis S et al (2002) Titania formation by $\mathrm{TiCl}_{4}$ gas phase oxidation, surface growth and coagulation. J Aerosol Sci 33:17-34

21. West RH, Celnik MS, Inderwildi OR et al (2007) Toward a comprehensive model of the synthesis of $\mathrm{TiO}_{2}$ particles from $\mathrm{TiCl}_{4}$. Ind Eng Chem Res 46:6147-6156 
22. West RH, Beran GJO, Green WH et al (2007) First-principles thermochemistry for the production of $\mathrm{TiO}_{2}$ from $\mathrm{TiCl}_{4}$. J Phys Chem A 111:3560-3565

23. Diebold U (2003) The surface science of titanium dioxide. Surf Sci Rep 48:53-229

24. Han N, Hu P, Zuo S (2010) Photoluminescence investigation on the gas sensing property of $\mathrm{ZnO}$ nanorods. Sens Actuators B 145:114-119

25. Chang PC, Fan ZY, Wang DW (2004) ZnO nanowires synthesized by vapor trapping CVD method. Chem Mater 16:5133-5137

26. Height MJ, Ma'dler L, Pratsinis SE (2006) Nanorods of ZnO made by flame spray pyrolysis. Chem Mater 18:572-578

27. Jood P, Mehta RJ, Zhang YL (2011) Al-doped zinc oxide nanocomposites with enhanced thermoelectric properties. Nano Lett 11:4337-4342

28. Remes Z, Vanecek M, Yates HM et al (2009) Optical properties of $\mathrm{SnO}_{2}: \mathrm{F}$ films deposited by atmospheric pressure CVD. Thin Solid Films 517:6287-6289

29. Vlahović B, Peršin M (1990) A simple and new modified CVD technique for fabrication of $\mathrm{SnO}_{2}$ films. J Phys D 23:1324-1326

30. Liu J, Gu F, Hu YJ et al (2010) Flame synthesis of tin oxide nanorods: a continuous and scalable approach. J Phys Chem C 114:5867-5870

31. Li Y, Wang H, Xie L et al (2011) $\mathrm{MoS}_{2}$ nanoparticles grown on graphene: an advanced catalyst for the hydrogen evolution reaction. J Am Chem Soc 133:7296-7299

32. Chianelli RR, Siadati MH, De la Rosa MP et al (2006) Catalytic properties of single layers of transition metal sulfide catalytic materials. Catal Rev 48:1-41

33. Gao MR, Jiang J, Yu SH (2012) Solution-based synthesis and design of late transition metal chalcogenide materials for oxygen reduction reaction (ORR). Small 8:13-27

34. Lee C, Li Q, Kalb W et al (2010) Frictional characteristics of atomically thin sheets. Science 328:76-80

35. Late DJ, Liu B, Matte HSSR et al (2012) Hysteresis in singlelayer $\mathrm{MoS}_{2}$ field effect transistors. ACS Nano 6:5635-5641

36. Radisavljevic B, Radenovic A, Brivio J et al (2011) Single-layer $\mathrm{MoS}_{2}$ transistors. Nat Nanotechnol 6:147-150

37. Lee HS, Min SW, Chang YG et al (2012) $\mathrm{MoS}_{2}$ nanosheet phototransistors with thickness-modulated optical energy gap. Nano Lett 12:3695-3700

38. Gao MR, Xu YF, Jiang J et al (2013) Nanostructured metal chalcogenides: synthesis, modification, and applications in energy conversion and storage devices. Chem Soc Rev 42:2986-3017

39. Lai CH, Lu MY, Chen LJ (2012) Metal sulfide nanostructures: synthesis, properties and applications in energy conversion and storage. J Mater Chem 22:19-30

40. Xu M, Liang T, Shi M et al (2013) Graphene-like two-dimensional materials. Chem Rev 113:3766-3798

41. Brito JL, Ilija M, Hernández P (1995) Thermal and reductive decomposition of ammonium thiomolybdates. Thermochim Acta 256:325-338

42. Shi Y, Zhou W, Lu AY et al (2012) Van der Waals epitaxy of $\mathrm{MoS}_{2}$ layers using graphene as growth templates. Nano Lett 12:2784-2791

43. Liu KK, Zhang W, Lee YH et al (2012) Growth of large-area and highly crystalline $\mathrm{MoS}_{2}$ thin layers on insulating substrates. Nano Lett 12:1538-1544

44. Helveg S, Lauritsen JV, Lægsgaard E et al (2000) Atomic-scale structure of single-layer $\mathrm{MoS}_{2}$ nanoclusters. Phys Rev Lett 84:951-954

45. Kibsgaard J, Lauritsen JV, Lægsgaard E et al (2006) Clustersupport interactions and morphology of $\mathrm{MoS}_{2}$ nanoclusters in a graphite-supported hydrotreating model catalyst. J Am Chem Soc 128:13950-13958
46. Jaramillo TF, Jorgensen KP, Bonde J et al (2007) Identification of active edge sites for electrochemical $\mathrm{H}_{2}$ evolution from $\mathrm{MoS}_{2}$ nanocatalysts. Science 317:100-102

47. Zhan Y, Liu Z, Najmaei S et al (2012) Large-area vapor-phase growth and characterization of $\mathrm{MoS}_{2}$ atomic layers on a $\mathrm{SiO}_{2}$ substrate. Small 8:966-971

48. Lee YH, Zhang XQ, Zhang W et al (2012) Synthesis of large-area $\mathrm{MoS}_{2}$ atomic layers with chemical vapor deposition. Adv Mater 24:2320-2325

49. Lee YH, Yu L, Wang H et al (2013) Synthesis and transfer of single-layer transition metal disulfides on diverse surfaces. Nano Lett 13:1852-1857

50. Shen G, Chen D, Chen PC et al (2009) Vapor-solid growth of one-dimensional layer-structured gallium sulfide nanostructures. ACS Nano 3:1115-1120

51. Ho CH, Lin SL (2006) Optical properties of the interband transitions of layered gallium sulfide. J Appl Phys 100:083508

52. Schlaf R, Armstrong NR, Parkinson BA et al (1997) Van der Waals epitaxy of the layered semiconductors $\mathrm{SnSe}_{2}$ and $\mathrm{SnS}_{2}$ : morphology and growth modes. Surf Sci 385:1-14

53. Li C, Huang L, Snigdha GP et al (2012) Role of boundary layer diffusion in vapor deposition growth of chalcogenide nanosheets: the case of GeS. ACS Nano 6:8868-8877

54. Peters ES, Carmalt CJ, Parkin IP (2004) Dual-source chemical vapour deposition of titanium sulfide thin films from tetrakisdimethylamidotitanium and sulfur precursors. J Mater Chem 14:3474-3477

55. Kong D, Wang H, Cha JJ et al (2013) Synthesis of $\mathrm{MoS}_{2}$ and $\mathrm{MoSe}_{2}$ films with vertically aligned layers. Nano Lett 13:1341-1347

56. Elias AL, Perea-Lopez N, Castro-Beltran A et al (2013) Controlled synthesis and transfer of large area $\mathrm{WS}_{2}$ sheets: from single-layer to few-layers. ACS Nano 7:5235-5242

57. Novak J, Šoltýs J, Eliáš P et al (2012) Electrical properties of individual GaP nanowires doped by zinc. Phys Status Solidi 209:2505-2509

58. Xiong Q, Gupta R, Adu KW et al (2003) Raman spectroscopy and structure of crystalline gallium phosphide nanowires. J Nanosci Nanotechnol 3:335-339

59. Gu ZJ, Paranthaman MP, Pan ZW (2009) Vapor-phase synthesis of gallium phosphide nanowires. Cryst Growth Des 9:525-527

60. Kim YH, Jun Y, Jun BH et al (2002) Sterically induced shape and crystalline phase control of GaP nanocrystals. J Am Chem Soc 124:13656-13657

61. Johansson J, Karlsson LS, Svensson CP et al (2006) Structural properties of $\langle 111\rangle$ B-oriented III-V nanowires. Nat Mater 5:574-580

62. Muskens OL, Diedenhofen SL, Kaas BC et al (2009) Large photonic strength of highly tunable resonant nanowire materials. Nano Lett 9:930-934

63. Borgström MT, Immink G, Ketelaars B et al (2007) Synergetic nanowire growth. Nat Nanotechnol 2:541-544

64. Borgström MT, Wallentin J, Ramvall P et al (2010) In situ etching for total control over axial and radial nanowire growth. Nano Res 3:264-270

65. Assali S, Zardo I, Plissard S et al (2013) Direct band gap wurtzite gallium phosphide nanowires. Nano Lett 13:1559-1563

66. Algra RE, Verheijen MA, Borgström MT et al (2008) Twinning superlattices in indium phosphide nanowires. Nature 456:369-372

67. Piret G, Perez MT, Prinz CN (2013) Neurite outgrowth and synaptophysin expression of postnatal CNS neurons on GaP nanowire arrays in long-term retinal cell culture. Biomaterials 34:875-887

68. Morales AM, Lieber CM (1998) A laser ablation method for the synthesis of crystalline semiconductor nanowires. Science 279:208-211 
69. Shi WS, Zheng YF, Wang N et al (2001) Synthesis and microstructure of gallium phosphide nanowires. J Vac Sci Technol B 19:1115-1118

70. Chen ZG, Cheng L, Lu GQ et al (2010) Sulfur-doped gallium phosphide nanowires and their optoelectronic properties. Nanotechnology 21:375701-375706

71. Wu Q, Hu Z, Liu C et al (2005) Synthesis and optical properties of gallium phosphide nanotubes. J Phys Chem B 109:19719-19722

72. Han W, Fan S, Li Q et al (1997) Synthesis of gallium nitride nanorods through a carbon nanotube-confined reaction. Science 277:1287-1289

73. Lin HM, Chen YL, Yang J et al (2003) Synthesis and characterization of core-shell GaP@GaN and GaN@GaP nanowires. Nano Lett 3:537-541

74. Fu LT, Chen ZG, Zou J et al (2010) Fabrication and visible emission of single-crystal diameter-modulated gallium phosphide nanochains. J Appl Phys 107:124321-124325
75. Ishizaka A, Shiraki Y (1986) Low temperature surface cleaning of silicon and its application to silicon MBE. J Electrochem Soc 133:666-671

76. Zou J, Paladugu M, Wang H et al (2007) Growth mechanism of truncated triangular III-V nanowires. Small 3:389-393

77. Heurlin M, Magnusson MH, Lindgren D et al (2012) Continuous gas-phase synthesis of nanowires with tunable properties. Nature 492:90-94

78. Gudiksen MS, Wang J, Lieber CM (2002) Size-dependent photoluminescence from single indium phosphide nanowires. J Phys Chem B 106:4036-4039

79. Ford AC, Ho JC, Chueh YL et al (2009) Diameter-dependent electron mobility of InAs nanowires. Nano Lett 9:360-365 